\title{
WEIGHTED BERGMAN KERNEL, DIRECTIONAL LELONG NUMBER AND JOHN-NIRENBERG EXPONENT
}

\author{
BO-YONG CHEN
}

\begin{abstract}
Let $\psi$ be a plurisubharmonic function on the closed unit ball and $K_{t \psi}(z)$ the Bergman kernel on the unit ball with respect to the weight $t \psi$. We show that the boundary behavior of $K_{t \psi}(z)$ is determined by certain directional Lelong number of $\psi$ for all $t$ smaller than the John-Nirenberg exponent of $\psi$ associated to certain family of nonisotropic balls, which is always positive.
\end{abstract}

KeYwords: Weighted Bergman kernel, plurisubharmonic function, directional Lelong number, John-Nirenberg exponent.

\section{INTRODUCTION}

Let $B_{1}$ be the unit ball in $\mathbb{C}^{n}$ and $P S H\left(B_{1}\right)$ the set of plurisubharmonic (psh) functions on $B_{1}$ (we always assume that psh functions are not identically $-\infty$ ). For each $\psi \in \operatorname{PSH}\left(B_{1}\right)$ we define $K_{t \psi}(z, w)$ to be the weighted Bergman kernel of the Hilbert space

$$
A_{t \psi}^{2}=\left\{f \in O\left(B_{1}\right): \int_{B_{1}}|f|^{2} e^{-t \psi}<\infty\right\}, \quad t \geq 0 .
$$

Set $K_{t \psi}(z)=K_{t \psi}(z, z)$. A cerebrated theorem of Demailly [6] states that

$$
\psi_{t}:=\frac{1}{t} \log K_{t \psi}(z) \rightarrow \psi(z) \quad(t \rightarrow+\infty)
$$

and

$$
v(\psi, z)-2 n / t \leq v\left(\psi_{t}, z\right) \leq v(\psi, z)
$$

where $v(\varphi, z)$ denotes the Lelong number for a psh function $\varphi$ at $z$.

In this paper we consider the case when $t$ is fixed and $z$ approaches the boundary $\partial B_{1}$. We discover that for all sufficiently small $t$ the asymptotic behavior of $K_{t \psi}(z)$ at a boundary point $\zeta$ is determined by certain directional Lelong number of $\psi$ at $\zeta$. To state the results precisely, we need to introduce some notions. Let

$$
\tilde{B}_{r}=\left\{\left(z_{1}, z^{\prime}\right) \in \mathbb{C} \times \mathbb{C}^{n-1}:\left|z_{1}\right|<r,\left|z^{\prime}\right|<\sqrt{r}\right\} .
$$

For a bounded domain $\Omega \subset \mathbb{C}^{n}$ we define $\tilde{\mathcal{B}}(\Omega)$ to be the set of all $F\left(\tilde{B}_{r}\right) \subset \Omega$ where $F$ is a complex affine mapping composed by a translation and a unitary transformation. We define the John-Nirenberg exponent of $\psi$ associated to the family $\tilde{\mathcal{B}}(\Omega)$ by

$$
\tilde{\varepsilon}_{\Omega}(\psi):=\sup \left\{\varepsilon: \sup _{D \in \tilde{\mathcal{B}}(\Omega)} f_{D} e^{\varepsilon\left|\psi-\psi_{D}\right|}<\infty\right\}
$$

where $\psi_{D}=f_{D} \psi$ is the mean value of $\psi$ over $D$. For each $\zeta \in \partial B_{1}$ we denote by $\mathcal{T}_{\zeta}$ the holomorphic tangent space at $\zeta$ and $\mathcal{N}_{\zeta}$ the orthogonal complement of $\mathcal{T}_{\zeta}$ in $\mathbb{C}^{n}$. Let $F_{\zeta}$ be the complex affine

Supported by NSF grant 11771089 and Gaofeng grant support from School of Mathematical Sciences, Fudan University. 
mapping which is composed by a translation and a unitary transformation, and maps the $z_{1}$ axis to $\mathcal{N}_{\zeta}$ and $z_{1}=0$ to $\mathcal{T}_{\zeta}$ respectively.

Theorem 1.1. Let $\psi$ be a psh function in a neighborhood of the closed ball $\bar{B}_{R}:=\{|z| \leq R\}$ where $R>1$. For each $0 \leq t<\tilde{\varepsilon}_{B_{R}}(\psi)$ and each $\zeta \in \partial B_{1}$ we have

$$
\lim _{r \rightarrow 0} \frac{\log K_{t \psi}((1-r) \zeta)}{\log 1 / r}=n+1-t \tilde{v}\left(\psi_{\zeta}\right)
$$

where $\psi_{\zeta}=\psi \circ F_{\zeta}$ and

$$
\tilde{v}\left(\psi_{\zeta}\right)=\lim _{r \rightarrow 0} \frac{1}{\log r} \sup _{\theta_{1}, \cdots, \theta_{n}} \psi_{\zeta}\left(r e^{i \theta_{1}}, \sqrt{r} e^{i \theta_{2}}, \cdots, \sqrt{r} e^{i \theta_{n}}\right) .
$$

Note that the quantity $2^{1-n} \tilde{v}\left(\psi_{\zeta}\right)$ is essentially the directional Lelong number with coefficients $(1,2, \cdots, 2)$ of $\psi_{\zeta}$ at 0 (see [7], p. 166).

Of course, Theorem 1.1 is meaningless unless one has verified the following

Theorem 1.2. Let $\Omega$ be a bounded domain in $\mathbb{C}^{n}$. If $\psi$ is psh in a neighborhood of $\bar{\Omega}$ then $\tilde{\varepsilon}_{\Omega}(\psi)>0$.

The proof of Theorem 1.2 relies on the following local Bernstein type inequality

$$
\sup _{B} \psi \leq \sup _{E} \psi+C_{n, \alpha}\left(1+\left|\psi_{B_{R}}\right|\right)^{\alpha}[1+\log (|B| /|E|)]
$$

for each ball $B \subset B_{R}$, measurable set $E \subset B$ and negative psh function $\psi$ on $B_{2 R}$, where $\alpha>$ 1 and $C_{n, \alpha}$ depends only on $n, \alpha$. Inequalities like (1.2) were obtained earlier by Brudnyi [3]. Analogous global inequalities were obtained by Benelkourchi et al. [1] for the Lelong class of psh functions. The analysis in these papers relies heavily on (nonlinear) pluripotential theory. Here we shall present an entirely new approach, using only linear analysis: the Riesz decomposition theorem and some basic facts from the theory of weights (see [14]).

\section{Proof of Theorem 1.1}

Theorem 1.1 will be deduced from the following

Theorem 2.1. Let $\psi$ be a psh function in a neighborhood of $\bar{B}_{R}$ where $R>1$. For each $0 \leq t<$ $\tilde{\varepsilon}_{B_{R}}(\psi)$ there exists a constant $C>0$ such that for all $\zeta \in \partial B_{1}$ and $0<r \ll 1$,

$$
C^{-1} r^{-n-1} e^{t \psi_{\tilde{B}_{r}(\zeta)}} \leq K_{t \psi}((1-r) \zeta) \leq C r^{-n-1} e^{t \psi_{\tilde{B}_{r}(\zeta)}}
$$

where $\tilde{B}_{r}(\zeta)=F_{\zeta}\left(\tilde{B}_{r}\right)$ and $F_{\zeta}$ is as in $\S 1$.

We start with a few elementary lemmas. For each $\zeta \in B_{1}$ we denote by $T_{\zeta}$ the holomorphic automorphism of $B_{1}$ which maps $\zeta$ onto the origin.

Lemma 2.2. Let $0<r<1$ and $\zeta_{r}:=(1-r, 0, \cdots, 0)$. Then we have

$$
\begin{gathered}
D_{r}:=\left\{z:\left|z_{1}-(1-r)\right|<r / 2,\left|z^{\prime}\right|<\sqrt{r / 2}\right\} \subset B_{1} \\
\left\{z:\left|T_{\zeta_{r}}(z)\right|<1 / \sqrt{2}\right\} \subset D_{r}^{\prime}:=\left\{z:\left|z_{1}-1\right|<10 r,\left|z^{\prime}\right|<\sqrt{20 r}\right\} .
\end{gathered}
$$

Proof. If $z \in D_{r}$, then $\left|z_{1}\right|<1-r / 2$, so that

$$
|z|^{2}<(1-r / 2)^{2}+r / 2=1-r / 2+r^{2} / 4<1,
$$

i.e. (2.2) is verified. 
Now suppose $\left|T_{\zeta_{r}}(z)\right|^{2}<1 / 2$. By the standard formula

$$
1-\left|T_{\zeta_{r}}(z)\right|^{2}=\frac{\left(1-|z|^{2}\right)\left(1-\left|\zeta_{r}\right|^{2}\right)}{\left|1-\left\langle z, \zeta_{r}\right\rangle\right|^{2}}
$$

(see [15], p. 5), we conclude that

$$
\begin{aligned}
2\left|1-z_{1}\right| & \geq 2\left(1-\left|z_{1}\right|\right) \geq 1-\left|z_{1}\right|^{2} \geq 1-\mid z^{2} \\
& \geq \frac{1}{2} \cdot \frac{\left|1-(1-r) z_{1}\right|^{2}}{2 r-r^{2}} \\
& \geq \frac{\left(\left|1-z_{1}\right|-r\left|z_{1}\right|\right)^{2}}{4 r-2 r^{2}} .
\end{aligned}
$$

With $c_{1}:=r\left|z_{1}\right|$ and $c_{2}:=4 r-2 r^{2}$ we have

$$
\left|1-z_{1}\right| \leq c_{1}+c_{2}+\sqrt{2 c_{1} c_{2}+c_{2}^{2}}<10 r .
$$

On the other hand, we have

$$
\left|z^{\prime}\right|^{2}<1-\left|z_{1}\right|^{2} \leq 2\left|1-z_{1}\right|<20 r
$$

Thus (2.3) is verified.

Lemma 2.3. Let $V$ be a measurable set in $\mathbb{R}^{n}$ and $\psi \in L^{1}(V)$. For each measurable set $W \subset V$ we have

$$
\begin{gathered}
\left|\psi_{W}-\psi_{V}\right| \leq \frac{|V|}{|W|} f_{V}\left|\psi-\psi_{V}\right| \\
f_{W}\left|\psi-\psi_{W}\right| \leq \frac{2|V|}{|W|} f_{V}\left|\psi-\psi_{V}\right| .
\end{gathered}
$$

Proof. First of all, we have

$$
\begin{aligned}
\left|\psi_{W}-\psi_{V}\right| & \leq \frac{1}{|W|} \int_{W}\left|\psi-\psi_{V}\right| \\
& \leq \frac{1}{|W|} \int_{V}\left|\psi-\psi_{V}\right| \\
& \leq \frac{|V|}{|W|} f_{V}\left|\psi-\psi_{V}\right|
\end{aligned}
$$

Next we have

$$
\begin{aligned}
f_{W}\left|\psi-\psi_{W}\right| & \leq f_{W}\left[\left|\psi-\psi_{V}\right|+\left|\psi_{W}-\psi_{V}\right|\right] \\
& \leq \frac{2|V|}{|W|} f_{V}\left|\psi-\psi_{V}\right| .
\end{aligned}
$$

Lemma 2.4. Let $\psi$ be a psh function in a neighborhood of $\bar{B}_{R}$ where $R>1$. For each $0 \leq t<\tilde{\varepsilon}_{B_{R}}(\psi)$ there exists a constant $C>0$ such that

$$
f_{D} e^{t \psi} \leq C e^{t \psi_{D}} \text { and } f_{D} e^{-t \psi} \leq C e^{-t \psi_{D}}
$$

for all $D \in \tilde{\mathcal{B}}\left(B_{R}\right)$. 
Proof. From the inequality

$$
f_{D} e^{t\left|\psi-\psi_{D}\right|} \leq C
$$

we immediately get (2.7).

Remark. By Jensen's inequality

$$
e^{t \psi_{D}} \leq f_{D} e^{t \psi} \text { and } e^{-t \psi_{D}} \leq f_{D} e^{-t \psi},
$$

one may call (2.7) the reverse Jensen inequality.

Proof of Theorem 2.1] Without loss of generality, we assume that $\zeta=(1,0, \cdots, 0)$. Set $\zeta_{r}=(1-r) \zeta$. For each $f \in A_{t \psi}^{2}$ we have

$$
\begin{aligned}
\left|f\left(\zeta_{r}\right)\right| \leq f_{D_{r}}|f| & \leq\left[f_{D_{r}}|f|^{2} e^{-t \psi}\right]^{1 / 2}\left[f_{D_{r}} e^{t \psi}\right]^{1 / 2} \\
& \leq\left[\int_{B_{1}}|f|^{2} e^{-t \psi}\right]^{1 / 2}\left[\frac{1}{\left|D_{r}\right|} f_{D_{r}} e^{t \psi}\right]^{1 / 2} \\
& \leq\left[\int_{B_{1}}|f|^{2} e^{-t \psi}\right]^{1 / 2}\left[C r^{-n-1} e^{t \psi_{D_{r}}}\right]^{1 / 2}
\end{aligned}
$$

where the last inequality follows from Lemma 2.4 . As

$$
\left|\psi_{D_{r}}-\psi_{\tilde{B}_{r}(\zeta)}\right| \leq C, \quad r \ll 1
$$

in view of Lemma 2.3 , we have

$$
K_{t \psi}\left(\zeta_{r}\right) \leq \sup _{f \in A_{t \psi}^{2}} \frac{\left|f\left(\zeta_{r}\right)\right|^{2}}{\|f\|_{r \psi}^{2}} \leq C r^{-n-1} e^{t \psi_{\tilde{B}_{r}(\zeta)}} .
$$

For the lower bound of $K_{t \psi}$ we shall use $L^{2}$-estimates of the $\bar{\partial}$-equation in a standard way (compare [5]). Let $g(z, w)$ be the pluricomplex Green function of $B_{1}$ with pole at $w$, i.e.

$$
g(z, w)=\log \left|T_{w}(z)\right|
$$

where $T_{w}$ is the holomorphic automorphism of $B_{1}$ which maps $w$ onto the origin. Set $g_{r}(z)=g\left(z, \zeta_{r}\right)$. Choose a smooth cut-off function $\chi: \mathbb{R} \rightarrow[0,1]$ such that $\left.\chi\right|_{(-\infty,-1]}=1$ and $\left.\chi\right|_{[0, \infty)}=0$. By the Donnelly-Fefferman estimate (see e.g. [2]), we may find a solution of

$$
\bar{\partial} u=\bar{\partial}\left[\chi\left(-\log \left(-g_{r}\right)+\log \log \sqrt{2}\right)\right]=: v
$$

which satisfies

$$
\begin{aligned}
\int_{B_{1}}|u|^{2} e^{-t \psi-2 n g_{r}} & \leq C_{0} \int_{B_{1}}|v|_{-i \partial \bar{\partial} \log \left(-g_{r}\right)}^{2} e^{-t \psi-2 n g_{r}} \\
& \leq C_{n} \int_{\left\{\left|T_{\zeta r}\right|<1 / \sqrt{2}\right\}} e^{-t \psi} \\
& \leq C_{n} \int_{D_{r}^{\prime}} e^{-t \psi}
\end{aligned}
$$

in view of Lemma2.2, where $C_{0}$ is a universal constant and $C_{n}$ depends only on $n$. Set

$$
f:=\chi\left(-\log \left(-g_{r}\right)+\log \log \sqrt{2}\right)-u .
$$


It follows that $f \in O\left(B_{1}\right), f\left(\zeta_{r}\right)=1$, and

$$
\int_{B_{1}}|f|^{2} e^{-t \psi} \leq C_{n} \int_{D_{r}^{\prime}} e^{-t \psi}
$$

Thus

$$
K_{t \psi}\left(\zeta_{r}\right) \geq \frac{\left|f\left(\zeta_{r}\right)\right|^{2}}{\|f\|_{t \psi}^{2}} \geq C_{n}^{-1}\left[\int_{D_{r}^{\prime}} e^{-t \psi}\right]^{-1} \geq C^{-1} r^{-n-1} e^{t \psi_{D_{r}^{\prime}}}
$$

in view of Lemma2.4. As

$$
\left|\psi_{D_{r}^{\prime}}-\psi_{\tilde{B}_{r}(\zeta)}\right| \leq C
$$

in view of Lemma 2.3, we establish the desired lower bound.

Proof of Theorem 1.1] Set $\tilde{P}_{r}=\left\{z:\left|z_{1}\right|<r, \max _{j \geq 2}\left|z_{j}\right|<\sqrt{r}\right\}$. Then we have

$$
\begin{aligned}
\liminf _{r \rightarrow 0} \psi_{\tilde{B}_{r}(\zeta)} / \log r & =\liminf _{r \rightarrow 0}\left(\psi_{\zeta}\right)_{\tilde{B}_{r}} / \log r \\
& =\liminf _{r \rightarrow 0}\left(\psi_{\zeta}\right)_{\tilde{P}_{r}} / \log r \\
& \geq \liminf _{r \rightarrow 0} \frac{1}{\log r} \sup _{\theta_{1}, \cdots, \theta_{n}} \psi_{\zeta}\left(r e^{i \theta_{1}}, r^{1 / 2} e^{i \theta_{2}}, \cdots, r^{1 / 2} e^{i \theta_{n}}\right) \\
& =\tilde{v}\left(\psi_{\zeta}\right)
\end{aligned}
$$

where the second equality follows from Lemma 2.3 and the inequality follows from the maximum principle for psh functions. On the other hand, for each $r$ we choose

$$
a_{r}=\left(r e^{i \theta_{1}(r)}, r^{1 / 2} e^{i \theta_{2}(r)}, \cdots, r^{1 / 2} e^{i \theta_{n}(r)}\right)
$$

such that

$$
\psi_{\zeta}\left(a_{r}\right)=\sup _{\theta_{1}, \cdots, \theta_{n}} \psi_{\zeta}\left(r e^{i \theta_{1}}, r^{1 / 2} e^{i \theta_{2}}, \cdots, r^{1 / 2} e^{i \theta_{n}}\right) .
$$

Set $\tilde{P}_{r}+a=\left\{z+a: z \in \tilde{P}_{r}\right\}$. By Lemma2.3 we have

$$
\begin{aligned}
\limsup _{r \rightarrow 0} \psi_{\tilde{B}_{r}(\zeta)} / \log r & =\limsup _{r \rightarrow 0}\left(\psi_{\zeta}\right)_{\tilde{B}_{r}} / \log r \\
& =\limsup _{r \rightarrow 0}\left(\psi_{\zeta}\right)_{\tilde{P}_{r}+a_{r}} / \log r \\
& \leq \limsup _{r \rightarrow 0} \psi_{\zeta}\left(a_{r}\right) / \log r \\
& =\tilde{v}\left(\psi_{\zeta}\right)
\end{aligned}
$$

where the inequality follows from the mean value inequality. Thus by (2.1) we establish (1.1).

\section{A local BMO estimate of psh functions}

A function $\psi \in L_{\text {loc }}^{1}(\Omega)$ is of BMO (bounded mean oscillation) if

$$
\|\psi\|_{\mathrm{BMO}(\Omega)}:=\sup _{B} f_{B}\left|\psi-\psi_{B}\right|<\infty,
$$

where the supremum is taken over all balls $B \subset \subset \Omega$. BMO was first introduced by John-Nirenberg [12] in connection with PDE, who also proved a crucial inequality:

$$
\sup _{B \subset \Omega} f_{B} e^{c_{n}\left|\psi-\psi_{B}\right| /|| \psi \|_{\mathrm{Bмо}(\Omega)}} \leq C_{n}
$$


where $c_{n}, C_{n}>0$ depend only on $n$. The BMO space became well-known after Fefferman proved that it is the dual of the real-variable Hardy space $H^{1}$ (cf. [9]). A famous unbounded example of $\operatorname{BMO}\left(\mathbb{R}^{n}\right)$ is $\log |x|$. We refer to Stein [14] for further examples and properties.

For a domain $\Omega \subset \mathbb{C}^{n}$ we define $\operatorname{PSH}^{-}(\Omega)$ to be the set of negative psh functions on $\Omega$. The purpose of this section is to show the following BMO estimate for psh functions.

Theorem 3.1. Let $\alpha>1$. If $\psi \in P S H^{-}\left(B_{2 R}\right)$, then

$$
\|\psi\|_{\mathrm{BMO}\left(B_{R}\right)} \leq C_{n, \alpha}\left(1+\left|\psi_{B_{R}}\right|\right)^{\alpha}
$$

where $C_{n, \alpha}>0$ depends only on $n, \alpha$.

Theorem 3.1 will be deduced from a number of lemmas.

Lemma 3.2. Let $\psi, \phi$ be two real $C^{2}$ function on a domain $\Omega \subset \mathbb{R}^{n}$. Let $\eta: \mathbb{R} \rightarrow(0, \infty)$ be a $C^{1}$ function with $\eta^{\prime}>0$. If either $\phi$ or $\psi$ has compact support in $\Omega$, then

$$
\int_{\Omega} \phi^{2}\left[\frac{2 \Delta \psi}{\eta(-\psi)}+\frac{\eta^{\prime}(-\psi)}{\eta^{2}(-\psi)}|\nabla \psi|^{2}\right] \leq 4 \int_{\Omega} \frac{|\nabla \phi|^{2}}{\eta^{\prime}(-\psi)} .
$$

Proof. Integration by parts gives

$$
\begin{aligned}
& \int_{\Omega} \frac{\phi^{2}}{\eta(-\psi)} \Delta \psi=-\int_{\Omega} \nabla \psi \cdot \nabla\left[\frac{\phi^{2}}{\eta(-\psi)}\right] \\
= & -2 \int_{\Omega} \phi \frac{\nabla \psi}{\eta(-\psi)} \cdot \nabla \phi-\int_{\Omega} \phi^{2} \frac{\eta^{\prime}(-\psi)}{\eta^{2}(-\psi)}|\nabla \psi|^{2},
\end{aligned}
$$

so that

$$
\begin{aligned}
& \int_{\Omega} \frac{\phi^{2}}{\eta(-\psi)} \Delta \psi+\int_{\Omega} \phi^{2} \frac{\eta^{\prime}(-\psi)}{\eta^{2}(-\psi)}|\nabla \psi|^{2} \\
= & -2 \int_{\Omega} \phi \frac{\nabla \psi}{\eta(-\psi)} \cdot \nabla \phi \\
\leq & \frac{1}{2} \int_{\Omega} \phi^{2} \frac{\eta^{\prime}(-\psi)}{\eta^{2}(-\psi)}|\nabla \psi|^{2}+2 \int_{\Omega} \frac{|\nabla \phi|^{2}}{\eta^{\prime}(-\psi)},
\end{aligned}
$$

from which (3.4) immediately follows.

Lemma 3.3. Let $\alpha>1$. If $\psi$ is a negative subharmonic function on a domain $\Omega \subset \mathbb{R}^{n}$, then

$$
\int_{\Omega} \phi^{2} \Delta \psi \leq \frac{4}{\alpha-1} \int_{\Omega}(1+|\psi|)^{\alpha}|\nabla \phi|^{2}, \quad \phi \in C_{0}^{\infty}(\Omega)
$$

Proof. We take a decreasing sequence of smooth subharmonic functions $\psi_{j}<0$ defined in a neighborhood of $\operatorname{supp} \phi$ such that $\psi_{j} \downarrow \psi$. Applying (3.4) with $\eta(t)=2-(1+t)^{1-\alpha}$, we have

$$
\int_{\Omega} \frac{\phi^{2}}{\eta\left(-\psi_{j}\right)} \Delta \psi_{j} \leq \frac{2}{\alpha-1} \int_{\Omega}\left(1+\left|\psi_{j}\right|\right)^{\alpha}|\nabla \phi|^{2} \leq \frac{2}{\alpha-1} \int_{\Omega}(1+|\psi|)^{\alpha}|\nabla \phi|^{2}
$$

As $\eta<2$, we have

$$
\begin{aligned}
\int_{\Omega} \phi^{2} \Delta \psi & =\lim _{j \rightarrow \infty} \int_{\Omega} \phi^{2} \Delta \psi_{j} \\
& \leq \frac{4}{\alpha-1} \int_{\Omega}(1+|\psi|)^{\alpha}|\nabla \phi|^{2} .
\end{aligned}
$$


Lemma 3.4. If $\psi \in S H^{-}(2 B)$, then

$$
\int_{2 B}|\psi| \leq 2^{n} \int_{B}|\psi|
$$

Proof. Let $\sigma_{n}$ be the volume of the unit sphere in $\mathbb{R}^{n}$. Write $B=B(a, r)$. Since $\psi$ is a subharmonic function, it follows that the mean value

$$
M_{\psi}(a, t)=\int_{|y|=1} \psi(a+t y) d \sigma(y) / \sigma_{n}
$$

is an increasing function of $t \in(0,2 r)$ (see [11], Theorem 3.2.3), i.e.

$$
M_{|\psi|}(a, t)=\int_{|y|=1}|\psi|(a+t y) d \sigma(y) / \sigma_{n}
$$

is a decreasing function of $t$. Then we have

$$
\begin{aligned}
\int_{2 B \backslash B}|\psi| & =\int_{r}^{2 r} M_{|\psi|}(a, t) t^{n-1} \sigma_{n} d t \\
& \leq\left(2^{n}-1\right) \frac{\sigma_{n}}{n} r^{n} M_{|\psi|}(a, r) \\
& \leq\left(2^{n}-1\right) \int_{0}^{r} M_{|\psi|}(a, t) t^{n-1} \sigma_{n} d t \\
& =\left(2^{n}-1\right) \int_{B}|\psi|,
\end{aligned}
$$

from which (3.5) immediately follows.

For the proof of Theorem 3.1 we consider at first the one-dimensional case.

Lemma 3.5. Let $\alpha>1$. If $n=1$ and $\psi \in S H^{-}\left(B_{2 R}\right)$, then

$$
f_{B}\left|\psi-\psi_{B}\right| \leq C_{\alpha} f_{B_{3 R / 2}}(1+|\psi|)^{\alpha}
$$

for all balls $B \subset B_{R}$. Here $C_{\alpha}>0$ depends only on $\alpha$.

Proof. Applying Lemma3.3 with $\phi \in C_{0}^{\infty}\left(B_{3 R / 2}\right)$ such that $\left.\phi\right|_{B_{4 R / 3}}=1$ and $|\nabla \phi| \leq 6 / R$, we conclude that

$$
\int_{B_{4 R / 3}} \Delta \psi \leq C_{\alpha} f_{B_{3 R / 2}}(1+|\psi|)^{\alpha} .
$$

Let $R^{\prime}=4 R / 3$. Recall that the (negative) Green function $g_{R^{\prime}}$ of $B_{R^{\prime}}$ is given by

$$
g_{R^{\prime}}(z, w)=\log |z-w|+\log \frac{R^{\prime}}{\left|R^{\prime 2}-z \bar{w}\right|} .
$$

The Riesz decomposition theorem (cf. [11], Theorem 3.3.6) gives

$$
\begin{aligned}
\psi(z) & =\frac{1}{2 \pi} \int_{\zeta \in B_{R^{\prime}}} g_{R^{\prime}}(z, \zeta) \Delta \psi(\zeta)+h(z) \\
& =\frac{1}{2 \pi} \int_{\zeta \in B_{R^{\prime}}} \log |z-\zeta| \Delta \psi(\zeta)+\frac{1}{2 \pi} \int_{\zeta \in B_{R^{\prime}}} \log \frac{R^{\prime}}{\left|R^{\prime 2}-z \bar{\zeta}\right|} \Delta \psi(\zeta)+h(z) \\
& =: \quad u(z)+v(z)+h(z)
\end{aligned}
$$

where $h$ is the smallest harmonic majorant of $\psi$, which naturally satisfies

$$
\psi \leq h \leq 0 .
$$


Since $h \leq 0$ is harmonic on $B_{R^{\prime}}$, it follows from the mean value property that for each $z \in B_{R}$

$$
\begin{aligned}
-h(z) & =f_{B(z, R / 4)}(-h) \\
& \leq \frac{1}{\pi(R / 4)^{2}} \int_{B_{R^{\prime}}}(-\psi) \\
& \leq C_{\alpha} f_{B_{3 R / 2}}(1+|\psi|)^{\alpha} .
\end{aligned}
$$

For each ball $B \subset B_{R}$ and $z \in B$, we have

$$
\begin{aligned}
2 \pi\left[u(z)-u_{B}\right] & =\int_{\zeta \in B_{R^{\prime}}} \log |z-\zeta| \Delta \psi(\zeta)-\frac{1}{|B|} \int_{w \in B} \int_{\zeta \in B_{R^{\prime}}} \log |w-\zeta| \Delta \psi(\zeta) \\
& =\int_{\zeta \in B_{R^{\prime}}}\left[\log |z-\zeta|-(\log |\cdot-\zeta|)_{B}\right] \Delta \psi(\zeta) .
\end{aligned}
$$

As the BMO norm on $\mathbb{C}^{n}$ is invariant under translations, it follows from Fubini's theorem that

$$
\begin{aligned}
f_{B}\left|u-u_{B}\right| & \leq \frac{1}{2 \pi}\|\log |z|\|_{\mathrm{BMO}\left(\mathbb{C}^{n}\right)} \int_{B_{R^{\prime}}} \Delta \psi \\
& \leq C_{\alpha} f_{B_{3 R / 2}}(1+|\psi|)^{\alpha} .
\end{aligned}
$$

Analogously, as

$$
\log \frac{R^{\prime}}{\left|R^{\prime 2}-z \bar{\zeta}\right|}=\log \frac{R^{\prime}}{|\zeta|}-\log \left|z-R^{\prime 2} / \bar{\zeta}\right|
$$

for $\zeta \neq 0$, it follows that the BMO norms (in $z$ ) of $\log \frac{R^{\prime}}{\left|R^{\prime 2}-z \zeta\right|}$ and $-\log |z|$ coincide, while for $\zeta=0$, $\log \frac{R^{\prime}}{\left|R^{\prime 2}-z \bar{\zeta}\right|} \equiv \log 1 / R^{\prime}$, so that its BMO norm is zero. Thus

$$
f_{B}\left|v-v_{B}\right| \leq C_{\alpha} f_{B_{3 R / 2}}(1+|\psi|)^{\alpha} .
$$

Clearly, (3.8)-(3.10) imply (3.6).

For each $a \in \mathbb{C}^{n}$ and each $\mathrm{r}=\left(r_{1}, \cdots, r_{n}\right)$ where $r_{j}>0$, we define the polydisc

$$
P(a, \mathrm{r})=\left\{z \in \mathbb{C}^{n}:\left|z_{j}-a_{j}\right|<r_{j}, 1 \leq j \leq n\right\} .
$$

Set $P(a, r)=P(a,(r, \cdots, r))$. Then we have

Lemma 3.6. Let $\alpha \geq 1$. If $\psi \in P S H^{-}(P(0,2 \mathrm{r}))$, then

$$
f_{P(0, \mathrm{r})}|\psi|^{\alpha} \leq C_{n, \alpha} f_{\left|z_{k}\right|<r_{k}}\left|\psi\left(0, \cdots, 0, z_{k}, 0, \cdots, 0\right)\right|^{\alpha}
$$

for all $1 \leq k \leq n$, where $C_{n, \alpha}>0$ depends only on $n, \alpha$.

Proof. It suffices to consider the case $k=1$. The Riesz decomposition theorem implies that if $u<0$ is a subharmonic function in a neighborhood of the unit closed disc in $\mathbb{C}$ then

$$
\int_{|z|<3 / 4}|u|^{\alpha} \leq C_{\alpha}|u(0)|^{\alpha}
$$


where $C_{\alpha}$ depends only on $\alpha$ (see [11], p. 230). In the case of $n$ complex variables we consider a negative psh function $u$ in a neighborhood of the unit closed polydisc in $\mathbb{C}^{n}$. Then we have

$$
\begin{aligned}
\int_{P(0,3 / 4)}|u|^{\alpha} & \leq C_{\alpha} \int_{\left|z_{1}\right|<3 / 4} \cdots \int_{\left|z_{n-1}\right|<3 / 4}\left|u\left(z_{1}, \cdots, z_{n-1}, 0\right)\right|^{\alpha} \\
& \leq \cdots \leq C_{\alpha}^{n-1} \int_{\left|z_{1}\right|<3 / 4}\left|u\left(z_{1}, 0^{\prime}\right)\right|^{\alpha},
\end{aligned}
$$

so that

$$
f_{P(0,3 / 4)}|u|^{\alpha} \leq C_{n, \alpha} f_{\left|z_{1}\right|<3 / 4}\left|u\left(z_{1}, 0^{\prime}\right)\right|^{\alpha} .
$$

It suffices to apply the above inequality with $u(z)=\psi\left(4 r_{1} z_{1} / 3, \cdots, 4 r_{n} z_{n} / 3\right)$.

Lemma 3.7. Let $\alpha>1$. If $\psi \in P S H^{-}(P(0,2 \mathrm{R}))$, then

$$
f_{P}\left|\psi-\psi_{P}\right| \leq C_{n, \alpha} \sum_{k=1}^{n} f_{\left|z_{k}\right|<3 R_{k} / 2}\left(1+\left|\psi\left(0, \cdots, 0, z_{k}, 0, \cdots, 0\right)\right|\right)^{\alpha}
$$

for any polydisc $P=P(0, \mathrm{r})$ with $\mathrm{r} \leq \mathrm{R}$, i.e. $r_{k} \leq R_{k}$ for all $k$.

Proof. We write $P=\prod_{j=1}^{n} B^{j}$ where $B^{j}=\left\{z_{j}:\left|z_{j}\right|<r_{j}\right\}$. For each $z \in P$ we have

$$
\begin{aligned}
\psi(z)-\psi_{P}= & \psi\left(z_{1}, z_{2}, \cdots, z_{n}\right)-\psi\left(\cdot, z_{2}, \cdots, z_{n}\right)_{B^{1}}+\cdots \\
& +\psi\left(\cdots, z_{k}, \cdots, z_{n}\right)_{B^{1} \cdots B^{k-1}}-\psi\left(\cdots, z_{k+1}, \cdots, z_{n}\right)_{B^{1} \cdots B^{k}}+\cdots \\
& +\psi\left(\cdots, z_{n}\right)_{B^{1} \cdots B^{n-1}}-\psi_{B^{1} \cdots B^{n}}
\end{aligned}
$$

where

$$
\psi\left(\cdots, z_{k}, \cdots, z_{n}\right)_{B^{1} \cdots B^{k-1}}=f_{\zeta_{1} \in B^{1}} \cdots f_{\zeta_{k-1} \in B^{k-1}} \psi\left(\zeta_{1}, \cdots, \zeta_{k-1}, z_{k} \cdots, z_{n}\right)
$$

Since

$$
\begin{aligned}
& \left|\psi\left(\cdots, z_{k}, \cdots, z_{n}\right)_{B^{1 \cdots} B^{k-1}}-\psi\left(\cdots, z_{k+1}, \cdots, z_{n}\right)_{B^{1} \cdots B^{k}}\right| \\
\leq & \left|\psi\left(\cdots, z_{k}, \cdots, z_{n}\right)-\psi\left(\cdots, z_{k+1}, \cdots, z_{n}\right)_{B^{k}}\right|_{B^{1 \cdots} B^{k-1}},
\end{aligned}
$$

it follows that

$$
\begin{aligned}
& f_{z_{k} \in B^{k}}\left|\psi\left(\cdots, z_{k}, \cdots, z_{n}\right)_{B^{1} \cdots B^{k-1}}-\psi\left(\cdots, z_{k+1}, \cdots, z_{n}\right)_{B^{1 \cdots} B^{k}}\right| \\
\leq & \left|f_{z_{k} \in B^{k}}\right| \psi\left(\cdots, z_{k}, \cdots, z_{n}\right)-\psi\left(\cdots, z_{k+1}, \cdots, z_{n}\right)_{B^{k}}||_{B^{1 \cdots B^{k-1}}} \\
\leq & C_{\alpha}\left[f_{\left|z_{k}\right|<3 R_{k} / 2}\left(1+\left|\psi\left(\cdots, z_{k}, \cdots, z_{n}\right)\right|\right)^{\alpha}\right]_{B^{1} \cdots B^{k-1}}
\end{aligned}
$$

in view of Lemma 3.5. Thus

$$
\begin{aligned}
& f_{P}\left|\psi-\psi_{P}\right| \leq C_{\alpha} \sum_{k=1}^{n}\left[f_{\left|z_{k}\right|<3 R_{k} / 2}\left(1-\psi\left(\cdots, z_{k}, \cdots\right)\right)^{\alpha}\right]_{B^{1} \cdots B^{k-1} B^{k+1} \cdots B^{n}} \\
& \leq C_{n, \alpha} \sum_{k=1}^{n} f_{\left|z_{k}\right|<3 R_{k} / 2}\left(1-\psi\left(0, \cdots, 0, z_{k}, 0, \cdots, 0\right)\right)^{\alpha}
\end{aligned}
$$

in view of Lemma 3.6, for $\psi-1$ is a negative psh function. 
Lemma 3.8. If $\psi \in P S H^{-}\left(B_{R}\right)$, then there exists $C_{n}>0$ depending only on $n$, such that there are complex lines $L_{1}, \cdots, L_{n}$ through the origin, which are orthogonal each other and satisfy $L_{j} \cap S \neq \emptyset$ for all $j$, where

$$
S=\left\{z \in B_{R / 3^{n}}: \psi(z)>C_{n} \psi_{B_{R / 3}}\right\}
$$

Proof. Set

$$
S_{m}=\left\{z \in B_{R / 3}: \psi(z)>m \psi_{B_{R / 3}}\right\}, \quad S_{m}^{c}=B_{R / 3}-S_{m} .
$$

By Chebychev's inequality

$$
\int_{B_{R / 3}}|\psi| \geq-m \psi_{B_{R / 3}}\left|S_{m}^{c}\right|
$$

we establish

$$
\left|S_{m}\right| \geq\left|B_{R / 3}\right|-\left|B_{R / 3}\right| / m>\left|B_{R / 3}\right| / 2
$$

provided $m>2$. We choose a universal constant $0<c_{1}<1 / 3$ such that

$$
\left|\left\{z \in B_{R / 3}:\left|z_{1}\right|<c_{1} R\right\}\right|+\left|\left\{z \in B_{R / 3}:\left|z_{2}\right|<c_{1} R\right\}\right|<\left|B_{R / 3}\right| / 4 .
$$

Set

$$
S_{m}^{\prime}=S_{m} \cap\left\{z: \min \left\{\left|z_{1}\right|,\left|z_{2}\right|\right\}>c_{1} R\right\}
$$

Then we have

$$
\left|S_{m}^{\prime}\right| \geq\left|S_{m}\right|-\left|B_{R / 3}\right| / 4>\left|S_{m}\right| / 2
$$

in view of (3.13). We define a diffeomorphism $F$ on $S_{m}^{\prime}$ as follows: $w_{j}=z_{j}$ for $j>1$ and

$$
w_{1}=-\left(\left|z_{2}\right|^{2}+\cdots+\left|z_{n}\right|^{2}\right) / \bar{z}_{1} .
$$

Clearly, the vector $F(z)$ is orthogonal to $z$ in $\mathbb{C}^{n}$ and satisfies

$$
|F(z)| \leq|z|^{2} /\left|z_{1}\right|<\left(9 c_{1}\right)^{-1} R,
$$

i.e. $\left(3 c_{1}\right) \cdot F(z) \in B_{R / 3}$. Since the real Jacobian $J_{\mathbb{R}}(F)$ of $F$ satisfies

$$
J_{\mathbb{R}}(F)(z)=-\left(\left|z_{2}\right|^{2}+\cdots+\left|z_{n}\right|^{2}\right)^{2} /\left|z_{1}\right|^{4}
$$

for $z \in S_{m}^{\prime}$, it follows that $\left|J_{\mathbb{R}}(F)(z)\right| \geq\left(3 c_{1}\right)^{4}$ and

$$
\left|\left(3 c_{1}\right) \cdot F\left(S_{m}^{\prime}\right)\right| \geq\left(3 c_{1}\right)^{2 n+4}\left|S_{m}^{\prime}\right|>\frac{1}{2}\left(3 c_{1}\right)^{2 n+4}\left|S_{m}\right|
$$

Thus if we choose

$$
m>\frac{1+\frac{1}{2}\left(3 c_{1}\right)^{2 n+4}}{\frac{1}{2}\left(3 c_{1}\right)^{2 n+4}}
$$

so that

$$
\left|S_{m}\right|>\left[1+\frac{1}{2}\left(3 c_{1}\right)^{2 n+4}\right]^{-1}\left|B_{R / 3}\right|
$$

in view of (3.13), then

$$
S_{m} \cap\left[\left(3 c_{1}\right) \cdot F\left(S_{m}^{\prime}\right)\right] \neq \emptyset .
$$

In other words, there exists a complex line $L_{1}$ such that both $L_{1}$ and its orthogonal complement $L_{1}^{\perp}$ in $\mathbb{C}^{n}$ intersect $S_{m}$. Suppose $a \in S_{m} \cap L_{1}^{\perp}$. The mean value inequality for the psh function $\psi$ implies

$$
f_{B(a, 2 R / 3) \cap L_{1}^{\perp}}|\psi| \leq|\psi(a)| .
$$


Since $B_{R / 3} \subset B(a, 2 R / 3)$, we have

$$
f_{B_{R / 3} \cap L_{1}^{\perp}}|\psi| \leq C_{n} m f_{B_{R / 3}}|\psi|
$$

where $C_{n}>0$ depends only on $n$. By repeating the previous argument, we obtain the remaining complex lines $L_{2}, \cdots, L_{n}$.

Proof of Theorem 3.1 Given $a \in B_{R}$, we have $B(a, R) \subset B_{2 R}$ and

$$
B(a, r) \subset P(a, r) \subset B(a, R / 3), \quad r \leq R /\left(3 n^{1 / 2}\right) .
$$

We assume $a=0$ for the sake of simplicity. Let $L_{j}, 1 \leq j \leq n$, be chosen as Lemma 3.8, By a unitary transformation, we may assume that $L_{j}$ is the $z_{j}$-axis for each $j$. By Lemma3.7 and Lemma 2.3 , we see that

$$
f_{B(0, r)}\left|\psi-\psi_{B(0, r)}\right| \leq C_{n, \alpha} \sum_{k=1}^{n} f_{\left|z_{k}\right|<R / 2}\left(1+\left|\psi\left(0, \cdots, 0, z_{k}, 0, \cdots, 0\right)\right|\right)^{\alpha} .
$$

Let $b^{(k)}=\left(0, \cdots, 0, b_{k}, 0, \cdots, 0\right) \in L_{k} \cap S$. It follows from (3.11) and Lemma 3.8 that

$$
f_{\left|z_{k}-b_{k}\right|<R / 2+R / 3^{n}}\left|\psi\left(0, \cdots, 0, z_{k}, 0, \cdots, 0\right)\right|^{\alpha} \leq C_{n, \alpha}\left|\psi\left(b^{(k)}\right)\right|^{\alpha} \leq C_{n, \alpha}\left|\psi_{B(0, R / 3)}\right|^{\alpha} .
$$

As

$$
\left\{z_{k}:\left|z_{k}\right|<R / 2\right\} \subset\left\{z_{k}:\left|z_{k}-b_{k}\right|<R / 2+R / 3^{n}\right\}
$$

we have

$$
f_{\left|z_{k}\right|<R / 2}\left|\psi\left(0, \cdots, 0, z_{k}, 0, \cdots, 0\right)\right|^{\alpha} \leq C_{n, \alpha}\left|\psi_{B(0, R / 3)}\right|^{\alpha} \leq C_{n, \alpha}\left|\psi_{B_{2 R}}\right|^{\alpha} \leq C_{n, \alpha}\left|\psi_{B_{R}}\right|^{\alpha}
$$

in view of Lemma 2.3 and Lemma 3.4. Thus

$$
f_{B(0, r)}\left|\psi-\psi_{B(0, r)}\right| \leq C_{n, \alpha}\left(1+\left|\psi_{B_{R}}\right|\right)^{\alpha} .
$$

On the other hand, for each ball $B(a, r) \subset B_{R}$ with $r>R /\left(3 n^{1 / 2}\right)$, we naturally have

$$
\begin{aligned}
f_{B(a, r)}\left|\psi-\psi_{B(a, r)}\right| & \leq 2\left|\psi_{B(a, r)}\right| \leq 2\left(1+\left|\psi_{B(a, r)}\right|\right)^{\alpha} \\
& \leq C_{n, \alpha}\left(1+\left|\psi_{B_{2 R}}\right|\right)^{\alpha} \\
& \leq C_{n, \alpha}\left(1+\left|\psi_{B_{R}}\right|\right)^{\alpha}
\end{aligned}
$$

in view of Lemma 2.3. Thus we have (3.3).

\section{Proof of Theorem 1.2}

Let us recall some basic facts from the theory of weights, by following Stein [14]. A local integrable function $\omega \geq 0$ on a domain $\Omega$ in $\mathbb{R}^{n}$ is said to satisfy the $A_{p}$ condition if

$$
\left[f_{B} \omega\right] \cdot\left[f_{B} \omega^{-1 /(p-1)}\right]^{p-1} \leq A<\infty
$$

for all balls $B \subset \subset \Omega$. The smallest constant $A$ for which (4.1) holds is called the $A_{p}$ constant of $\omega$, which is denoted by $A_{p}(\omega)$. It is known that $\omega \in A_{p}$ if and only if

$$
\left(f_{B}\right)^{p} \leq C\left[\int_{B} f^{p} \omega\right] \cdot\left[\int_{B} \omega\right]^{-1}
$$


for all nonnegative $f \in L_{\text {loc }}^{1}(\Omega)$ and all balls $B \subset \subset \Omega$; moreover the smallest $C$ for which (4.2) is valid equals $A_{p}(\omega)$ (see [14], p. 195). Let $E$ be a measurable set in $B$ and $\chi_{E}$ the characteristic function of $E$. Applying (4.2) with $f=\chi_{E}$ we establish

$$
\int_{B} \omega \leq A_{p}(\omega)(|B| /|E|)^{p} \int_{E} \omega
$$

In particular, $\omega$ satisfies a doubling property

$$
\int_{B} \omega \leq 2^{n p} A_{p}(\omega) \int_{\frac{1}{2} B} \omega .
$$

Let $\psi \in \operatorname{BMO}(\Omega)$ and $u:=c_{n} \psi /\|\psi\|_{\mathrm{BMO}(\Omega)}$, where $c_{n}$ is the constant in (3.2). Then we have

$$
f_{B} e^{u-u_{B}} \leq C_{n}, \quad f_{B} e^{u_{B}-u} \leq C_{n},
$$

so that

$$
\left[f_{B} e^{u}\right] \cdot\left[f_{B} e^{-u}\right]=\left[f_{B} e^{u-u_{B}}\right] \cdot\left[f_{B} e^{u_{B}-u}\right] \leq C_{n}^{2},
$$

i.e. $e^{u}, e^{-u} \in A_{2}$. Applying (4.3) with $\omega=e^{u}$ we establish

$$
\int_{B} e^{u} \leq C_{n}(|B| /|E|)^{2} \int_{E} e^{u}
$$

Now we can prove the following inequality mentioned in $\S 1$.

Theorem 4.1. Let $B_{R}=\left\{z \in \mathbb{C}^{n}:|z|<R\right\}$ and $\alpha>1$. If $\psi \in P S H^{-}\left(B_{2 R}\right)$, then for each ball $B \subset B_{R}$ and each measurable set $E \subset B$ one has

$$
\sup _{B} \psi \leq \sup _{E} \psi+C_{n, \alpha}\left(1+\left|\psi_{B_{R}}\right|\right)^{\alpha}[1+\log (|B| /|E|)]
$$

where $C_{n, \alpha}>0$ depends only on $n, \alpha$.

Proof. Let $B$ be a ball in $B_{R}$ and $E$ a measurable set in $B$. Then we have

$$
\int_{E} e^{u} \leq|E| e^{\sup _{E} u}
$$

where $u=c_{n} \psi /\|\psi\|_{\mathrm{BMO}\left(B_{R}\right)}$. On the other hand, we choose a point $a \in \bar{B}$ such that $u(a)=\sup _{B} u$. Let $r$ be the radius of $B$. The doubling property (4.4) implies

$$
\int_{B} e^{u} \geq C_{n}^{-1} \int_{2 B} e^{u} \geq C_{n}^{-1} \int_{B(a, r)} e^{u} \geq C_{n}^{-1}|B(a, r)| e^{u(a)} \geq C_{n}^{-1}|B| e^{\sup _{B} u}
$$

where the third inequality follows from the mean value inequality for the psh function $e^{u}$. Combining (4.6), (4.8) and (4.9) yields

$$
\sup _{B} u \leq \sup _{E} u+\log (|B| /|E|)+C_{n} .
$$

This inequality combined with Theorem 3.1 gives (4.7).

Theorem 4.1 implies a new interpretation of the Lelong number.

Corollary 4.2. Let $\psi$ be a psh function on a domain $\Omega \subset \mathbb{C}^{n}$. Let $v(\psi, z)$ denote the Lelong number of $\psi$ at $z \in \Omega$. Let $E_{r}, 0<r \ll 1$, be a family of measurable sets satisfying $E_{r} \subset B(z, r)$ and

$$
\log \left(|B(z, r)| /\left|E_{r}\right|\right)=\mathrm{o}(\log 1 / r), \quad r \rightarrow 0 .
$$


Then we have

$$
v(\psi, z)=\lim _{r \rightarrow 0}\left(\sup _{E_{r}} \psi\right) / \log r
$$

Proof. After subtracting a constant to $\psi$ we may assume $\psi<0$ on $B\left(z, r_{0}\right)$ for some $r_{0}<d(z, \partial \Omega)$. As

we have

$$
v(\psi, z)=\lim _{r \rightarrow 0}\left(\sup _{B(z, r)} \psi\right) / \log r
$$

$$
v(\psi, z) \leq \liminf _{r \rightarrow 0}\left(\sup _{E_{r}} \psi\right) / \log r .
$$

On the other hand, (4.7) implies

$$
v(\psi, z) \geq \limsup _{r \rightarrow 0}\left(\sup _{E_{r}} \psi\right) / \log r .
$$

Theorem 4.3. Let $\alpha>2$ and $\gamma>1$. If $\psi \in \operatorname{PSH}^{-}\left(\tilde{B}_{2 R}\right)$, then there exist positive constants $c_{n, \alpha, \gamma}$ depends only on $n, \alpha, \gamma$ and $C_{n}$ depending only on $n$ such that

$$
f_{\tilde{B}_{r}+a} e^{\varepsilon\left|\psi-\psi_{\tilde{B}_{r}+a}\right|} \leq C_{n}
$$

for all $\tilde{B}_{r}+a:=\left\{z+a: z \in \tilde{B}_{r}\right\} \subset \tilde{B}_{R}$, where

$$
\varepsilon=c_{n, \alpha, \gamma}\left[1+f_{\tilde{B}_{3 R / 2}}|\psi|^{\alpha}\right]^{-\gamma / \alpha} .
$$

Let us first observe that Theorem 1.2 follows from Theorem 4.3 , Let $U$ be a neighborhood of $\bar{\Omega}$ such that $\psi$ is psh on $\bar{U}$. Let $D \in \tilde{\mathcal{B}}(\Omega)$. By a change of complex coordinates we may assume that $D$ is of form $\tilde{B}_{r}$ for some $r>0$. As $\tilde{B}_{2 R} \subset U$ for $R:=d(D, \partial U)^{2} / 4$ (assume $d(D, \partial U) \leq 1$ for the sake of simplicity), we apply Theorem 4.3 with $\psi$ replaced by $\tilde{\psi}:=\psi-\sup _{U} \psi$ to get

$$
f_{D} e^{\varepsilon\left|\psi-\psi_{D}\right|} \leq C_{n}
$$

provided

$$
\begin{aligned}
\varepsilon & =c_{n, \alpha, \gamma}\left[1+f_{\tilde{B}_{3 R / 2}}|\tilde{\psi}|^{\alpha}\right]^{-\gamma / \alpha} \\
& \geq c_{n, \alpha, \gamma}\left[1+d(D, \partial U)^{-2(n+1)} \int_{U}|\tilde{\psi}|^{\alpha}\right]^{-\gamma / \alpha} .
\end{aligned}
$$

This completes the proof of Theorem 1.2 .

Theorem 4.3 will be deduced from the following inequalities.

Lemma 4.4. Let $\alpha>2$ and $\gamma>1$. If $\psi \in P S H^{-}\left(\tilde{B}_{2 R}\right)$, then there exists a number

$$
0<\lambda \leq C_{n, \alpha, \gamma}\left[1+f_{\tilde{B}_{3 R / 2}}|\psi|^{\alpha}\right]^{\gamma / \alpha}
$$

such that for each $r \leq R / 2$ and $a$ with $\tilde{B}_{r}+a \subset \tilde{B}_{R}$,

$$
\begin{gathered}
{\left[f_{\tilde{B}_{r}+a} e^{2 \psi / \lambda}\right]^{1 / 2} \leq C_{n} f_{\tilde{B}_{r}+a} e^{\psi / \lambda}} \\
\int_{\tilde{B}_{r}+a} e^{\psi / \lambda} \leq C_{n} \int_{\tilde{B}_{r / 2}+a} e^{\psi / \lambda} .
\end{gathered}
$$


Proof. For each $r$ and $a$ with $\tilde{B}_{r}+a \subset \tilde{B}_{R}$ we define $R_{a, r}$ to be the supremum of all $t \geq r$ such that

$$
\tilde{B}_{t}+a \subset \tilde{B}_{3 R / 2} \text {. }
$$

Clearly we have $c_{n} R \leq R_{a, r} \leq 3 R / 2$ and

$$
f_{\tilde{B}_{R a, r}+a}|\psi|^{\alpha} \leq C_{n} f_{\tilde{B}_{3 R / 2}}|\psi|^{\alpha} .
$$

It suffices to verify (4.13) and (4.14) with

$$
\lambda \leq C_{n, \alpha, \gamma}\left[1+f_{\tilde{B}_{R_{a, r}+a}}|\psi|^{\alpha}\right]^{\gamma / \alpha} .
$$

For the sake of simplicity we assume $a=0$ and write $R_{a, r}$ as $R$. Set $\varphi(\zeta)=\psi\left(\zeta_{1}^{2}, \zeta^{\prime}\right)$ and

$$
B_{r}^{*}=\left\{\zeta \in \mathbb{C}^{n}:\left|\zeta_{1}\right|<r,\left|\zeta^{\prime}\right|<r\right\} .
$$

Let $r^{\prime}=\sqrt{r}$ and $\gamma>1$. By Theorem 4.1 we conclude that if $r^{\prime} \leq R^{\prime} / \sqrt{2}$ then

$$
\begin{aligned}
& \sup _{B_{r^{\prime}}^{*}} \varphi \leq \sup _{B\left(0, \sqrt{2} r^{\prime}\right)} \varphi \leq \sup _{B_{r^{\prime} / 2}^{*}} \varphi+C_{n, \gamma}\left(1+|\varphi|_{B\left(0, R^{\prime}\right)}\right)^{\gamma}\left[1+\log \frac{\left|B\left(0, \sqrt{2} r^{\prime}\right)\right|}{\left|B_{r^{\prime} / 2}^{*}\right|}\right] \\
& \leq \sup _{B_{r^{\prime} / 2}^{*}} \varphi+C_{n, \gamma}\left(1+|\varphi|_{B_{R^{\prime}}^{*}}\right)^{\gamma} \\
& =: \sup _{B_{r^{\prime} / 2}^{*}} \varphi+\lambda \text {. }
\end{aligned}
$$

It follows that

$$
\sup _{B_{r^{\prime}}^{*}} e^{\varphi / \lambda} \leq e \sup _{B_{r^{\prime} / 2}^{*}} e^{\varphi / \lambda}
$$

i.e.

$$
\sup _{\tilde{B}_{r}} e^{\psi / \lambda} \leq e \sup _{\tilde{B}_{r / 4}} e^{\psi / \lambda}
$$

Then we have

$$
\begin{gathered}
{\left[f_{\tilde{B}_{r}} e^{2 \psi / \lambda}\right]^{1 / 2} \leq \sup _{\tilde{B}_{r}} e^{\psi / \lambda} \leq e \sup _{\tilde{B}_{r / 4}} e^{\psi / \lambda} \leq C_{n} f_{\tilde{B}_{r}} e^{\psi / \lambda}} \\
f_{\tilde{B}_{r}} e^{2 \psi / \lambda} \leq \sup _{\tilde{B}_{r}} e^{\psi / \lambda} \leq e \sup _{\tilde{B}_{r / 4}} e^{\psi / \lambda} \leq C_{n} f_{\tilde{B}_{r / 2}} e^{\psi / \lambda}
\end{gathered}
$$

in view of the mean value inequality for the psh function $e^{\psi / \lambda}$.

Let $\alpha^{\prime}$ be the dual exponent of $\alpha$. As

$$
\begin{aligned}
f_{B_{R^{\prime}}^{*}}|\varphi| & \leq \frac{1}{\left|B_{R^{\prime}}^{*}\right|}\left[\int_{B_{R^{\prime}}^{*}}\left|\zeta_{1}\right|^{-2 \alpha^{\prime} / \alpha}\right]^{1 / \alpha^{\prime}}\left[\int_{B_{R^{\prime}}^{*}}|\varphi|^{\alpha}\left|\zeta_{1}\right|^{2}\right]^{1 / \alpha} \\
& =\frac{1}{\left|B_{R^{\prime}}^{*}\right|}\left[\int_{B_{R^{\prime}}^{*}}\left|\zeta_{1}\right|^{-2 \alpha^{\prime} / \alpha}\right]^{1 / \alpha^{\prime}}\left[\frac{1}{2} \int_{\tilde{B}_{R}}|\psi|^{\alpha}\right]^{1 / \alpha} \\
& \leq C_{n, \alpha} R^{-(n+1) / \alpha}\left[\int_{\tilde{B}_{R}}|\psi|^{\alpha}\right]^{1 / \alpha} \\
& \leq C_{n, \alpha}\left[\int_{\tilde{B}_{R}}|\psi|^{\alpha}\right]^{1 / \alpha},
\end{aligned}
$$


we have

$$
\lambda \leq C_{n, \alpha, \gamma}\left[1+f_{\tilde{B}_{R}}|\psi|^{\alpha}\right]^{\gamma / \alpha} .
$$

Proof of Theorem 4.3 It is a standard fact that the reverse Hölder inequality like (4.13) and the doubling property like (4.14) for Euclidean balls would imply the $A_{p}$ property for some $p>1$. We shall show that the same is true for nonisotropic balls $\tilde{B}_{r}+a$ by using Calderón's work [4]. We define

$$
\varrho(z, w)=\max \left\{\left|z_{1}-w_{1}\right|,\left|z^{\prime}-w^{\prime}\right|^{2}\right\}, \quad z, w \in \mathbb{C}^{n} .
$$

It is easy to verify that $\varrho$ satisfies the following properties

(1) $\varrho(z, z)=0$;

(2) $\varrho(z, w)=\varrho(w, z)>0$ if $z \neq w$;

(3) $\varrho(z, w) \leq 2(\varrho(z, \zeta)+\varrho(\zeta, w))$ for all $z, w, \zeta \in \mathbb{C}^{n}$.

Note also that $\tilde{B}_{r}+a=\{z: \varrho(z, a)<r\}$. Set $\omega=e^{\psi / \lambda}$ and $d \mu=\omega d V$ where $d V$ is the Lebesgue measure in $\mathbb{C}^{n}$. Let $|\cdot|_{\mu}$ be the volume associated to $d \mu$. Then we may rewrite (4.14) as

$$
\left|\tilde{B}_{r}+a\right|_{\mu} \leq C_{n}\left|\tilde{B}_{r / 2}+a\right|_{\mu} .
$$

Let $E$ be a measurable set in $\tilde{B}_{r}+a$. By (4.13) we have

$$
\begin{aligned}
|E|_{\mu}=\int_{E} \omega & \leq\left[\int_{E} \omega^{2}\right]^{1 / 2}|E|^{1 / 2} \\
& \leq\left[\int_{\tilde{B}_{r}+a} \omega^{2}\right]^{1 / 2}|E|^{1 / 2} \\
& \leq C_{n}\left|\tilde{B}_{r}+a\right|^{-1 / 2}\left[\int_{\tilde{B}_{r}+a} \omega\right]|E|^{1 / 2},
\end{aligned}
$$

i.e.

$$
\frac{|E|}{\left|\tilde{B}_{r}+a\right|} \geq C_{n}^{-1}\left[\frac{|E|_{\mu}}{\left|\tilde{B}_{r}+a\right|_{\mu}}\right]^{2} .
$$

According to Calderón (see [4], the proof of Theorem 1), the above inequality implies a reverse Hölder inequality w.r.t. the measure $d \mu$ (noting that $d V=\omega^{-1} d \mu$ )

$$
\left[\frac{1}{\left|\tilde{B}_{r}+a\right|_{\mu}} \int_{\tilde{B}_{r}+a} \omega^{-p_{n}} d \mu\right]^{1 / p_{n}} \leq C_{n} \frac{1}{\left|\tilde{B}_{r}+a\right|_{\mu}} \int_{\tilde{B}_{r}+a} \omega^{-1} d \mu,
$$

for some $p_{n}>1$, which may be rewritten as

$$
\left[f_{\tilde{B}_{r}+a} \omega\right] \cdot\left[f_{\tilde{B}_{r}+a} \omega^{-p_{n}+1}\right]^{1 /\left(p_{n}-1\right)} \leq C_{n} .
$$

It follows that

$$
\left[f_{\tilde{B}_{r}+a} e^{\left(\psi-\psi_{\tilde{B}_{r}+a}\right) / \lambda}\right] \cdot\left[f_{\tilde{B}_{r}+a} e^{-\left(p_{n}-1\right)\left(\psi-\psi_{\tilde{B}_{r}+a}\right) / \lambda}\right]^{1 /\left(p_{n}-1\right)} \leq C_{n} .
$$

This inequality combined with Jensen's inequality

$$
f_{\tilde{B}_{r}+a} e^{\left(\psi-\psi_{\tilde{B}_{r}+a}\right) / \lambda} \geq 1 \text { and } f_{\tilde{B}_{r}+a} e^{-\left(p_{n}-1\right)\left(\psi-\psi_{\tilde{B}_{r}+a}\right) / \lambda} \geq 1
$$


yields

$$
f_{\tilde{B}_{r}+a} e^{\left(\psi-\psi_{\tilde{B}_{r}+a}\right) / \lambda} \leq C_{n} \text { and } f_{\tilde{B}_{r}+a} e^{-\left(p_{n}-1\right)\left(\psi-\psi_{\tilde{B}_{r}+a}\right) / \lambda} \leq C_{n} .
$$

Set $u=\left(\psi-\psi_{\tilde{B}_{r}+a}\right) / \lambda$ and $\varepsilon_{n}=\min \left\{1, p_{n}-1\right\}$. Then we have

$$
\begin{aligned}
f_{\tilde{B}_{r}+a} e^{\varepsilon_{n}\left|\psi-\psi_{\tilde{B}_{r}+a}\right| / \lambda} & =\frac{1}{\left|\tilde{B}_{r}+a\right|} \int_{\{u \geq 0\}} e^{\varepsilon_{n} u}+\frac{1}{\left|\tilde{B}_{r}+a\right|} \int_{\{u<0\}} e^{-\varepsilon_{n} u} \\
& \leq f_{\tilde{B}_{r}+a} e^{u}+f_{\tilde{B}_{r}+a} e^{-\left(p_{n}-1\right) u} \\
& \leq C_{n} .
\end{aligned}
$$

\section{REFERENCES}

[1] S. Benelkourchi, B. Jennane and A. Zeriahi, Polya's inequalities, global uniform integrability and the size of plurisubharmonic lemniscates, Ark. Mat. 43 (2005), 85-112.

[2] B. Berndtsson and Ph. Charpentier, A Sobolev mapping property of the Bergman kernel, Math. Z. 235 (2000), 1-10.

[3] A. Brudnyi, Local inequalities for plurisubharmonic functions, Ann. of Math. 149 (1999), 511-533.

[4] A. P. Calderón, Inequalities for the maximal function relative to a metric, Studia Math. 57 (1976), 297-306.

[5] B.-Y. Chen, Completeness of the Bergman metric on non-smooth pseudoconvex domains, Ann. Polon. Math. 71 (1999), 241-251.

[6] J.-P. Demailly, Regularization of closed positive currents and Intersection Theory, J. Alg. Geom. 1 (1992), $361-409$.

[7] J.-P. Demailly, Complex Analytic and Differential Geometry, available at Demailly's home page.

[8] J.-P. Demailly and J. Kollár, Semi-continuity of complex singularity exponents and Kähler-Einstein metrics on Fano orbifolds, Ann. Scient. Éc. Norm. Sup. 34 (2001), 525-556.

[9] C. Fefferman and E. M. Stein, $H^{p}$ spaces of several variables, Acta Math. 129 (1972), 137-193.

[10] L. Hörmander, An introduction to Complex Analysis in Several Variables, North Holland, 1990.

[11] L. Hörmander, Notions of convexity, Birkhäuser, 1994.

[12] F. John and L. Nirenberg, On functions of bounded mean oscillation, Comm. Pure Appl. Math. 14 (1961), 415-426.

[13] H. Skoda, Sous-ensembles analytiques d'ordre fini ou infini dans $\mathbb{C}^{n}$, Bull. Soc. Math. France 100 (1972), $353-408$.

[14] E. M. Stein, Harmonic Analysis: Real-Variable Methods, Orthogonality, and Oscillatory Integrals, Princeton Univ. Press, Princeton, New Jersey, 1993.

[15] K. Zhu, Spaces of Holomorphic Functions in the Unit Ball, GTM 226, Springer Science+Business Media, 2005.

School of Mathematical Sciences, Fudan University, Shanghai, 200433, China

E-mail address: boychen@fudan. edu.cn 